\title{
Cybernetics of the Collaborative Networks Discipline
}

\author{
Hadi Kandjani and Peter Bernus \\ Centre for Enterprise Architecture Research and Management (CEARM) \\ School of ICT, Griffith University, Brisbane, Australia \\ $\{$ H.Kandjani, P.Bernus\}@griffith.edu.au
}

\begin{abstract}
Collaborative Networks (CNs) research, like any other developing discipline, needs a roadmap facilitating the integration of previous results into a theoretical foundation. $\mathrm{CN}$ researchers argue that the required theoretical foundation must consolidate the existing body of knowledge, and provide grounding to define how to invoke results of other relevant disciplines. The authors have previously proposed the 'Cybernetics of the Collaborative Networks' (C2N) as a field of CN-research intended as a unified theory of CNs, formalising, synthesising, harmonising and systematising individual $\mathrm{CN}$-related results addressing management and control problems in CNs. This article aims at further extending the concept of $\mathrm{C} 2 \mathrm{~N}$ and answering the question: what is a unified evolving theory of the $\mathrm{CN}$ discipline itself? To model the discipline-asa-system, we use Beer's Viable System Model (VSM) and introduce three basic components of the $\mathrm{CN}$ discipline as a viable system. A 'co-evolution mechanisms' for the discipline is proposed and a cybernetic model of coevolution is applied to the $\mathrm{CN}$ discipline.
\end{abstract}

Keywords: Collaborative Networks, Cybernetics, Unified Developing Theory, Evolving Discipline.

\section{Introduction}

'Cybernetics of the Collaborative Networks (C2N)' was previously proposed as a field of research intended to address complexity management and control problems in $\mathrm{CNs}$ [1]. C2N can be thought of a field on its own, which re-interprets old and new theories, and point at the need for genuinely new results for designing / creating and managing complex CNs. The aim of this previous research was to understand how various theories contribute to the management of CNs, so that the Network evolves and remains viable in the long term. As part of this endeavor, the authors developed a model (called the co-evolution path model), discussing the evolution of a $\mathrm{CN}$ in light of the evolution of the Network's environment, so as the Network can remain relevant, competitive, and viable.

The idea of this paper is to look at the evolution of the discipline itself, i.e., how the discipline of CNs evolves? After all, a discipline can also be considered a system (of concepts, axioms, theories, scope definitions, paradigms, etc.), and it is relevant to ask: how does discipline development ensure that it remains relevant on the long 
term? What we set out to discuss, is a theory of the evolution of the $\mathrm{CN}$ body of knowledge. This theory is to describe the evolution mechanisms of the $\mathrm{CN}$ body of knowledge, but is open for further development by $\mathrm{CN}$ practitioners and researchers.

The or interdisciplinary aspect of CNs manifests when researchers not only apply models, methods and theories of management and control, engineering, linguistics, cognitive science, environmental science, biology, social science, artificial intelligence, systems thinking and cybernetics, but also create a synthesis of these. Such a synthesis would be the source of a new, unified theory, giving rise to more powerful theories, methodologies and reference models than available today.

For the study of Collaborative Networks, previous work has developed a roadmap to facilitate the invocation and integration of previous results into a theoretical foundation, including terminology, axioms, models, and methodologies $[2,3,4,5,6,7,8,9]$.

The critical question in the present paper is: is it possible to develop a theory of how the CN Body of Knowledge is developing as a discipline?

Kandjani and Bernus [1] argue that Cybernetics [10,11], Management Cybernetics $[12,13]$ and General Systems Theory (GST) [14,15] have previously tackled these types of problems at the same, or similar, level of abstraction and generality, therefore, to describe discipline evolution we use a similar approach to the cybernetics-inspired discussion of how CNs develop.

\section{Viability of the CN Discipline and Effective CN Practice}

In this section we propose a viable model of the $\mathrm{CN}$ body of knowledge, as an interdisciplinary discipline of designing, creating and maintaining CNs.

\subsection{Beer's Viable System Model}

Beer [16] describes every system as consisting of three main interacting components: Management, Operation and Environment. Every system of interest has a metasystem as its management and operates in an environment, where each component could be further decomposed into more detailed elements. There are communication channels to keep the Operation in homeostasis, these channels are called variety attenuators and -amplifiers $[16,17,13]$, to ensure that the variety what the Operations can provide meets the variety of the demands of the environment.

According to Beer [16] the 'variety' of the operations is always less than that of the Environment, and the 'variety of Management' is always less than the variety of Operations. In contrast, based on Ashby's law of requisite variety (1956), in order to achieve dynamic stability under change, the variety of Operations should be equal to that of its relevant environment, and the variety of Management should be at least equal to that of Operations. In fact variety attenuation and amplification mechanisms need to be designed in order to keep the system of interest viable ('evolvable') in its environment. 


\subsection{The CN Discipline as a Viable System}

One can map the three components of Beer's VSM to the CN Discipline itself, and to its surrounding Environment (Fig. 1). We consider CN-related disciplines as 'Operations' shown as a circle, and the $\mathrm{CN}$ discipline as its integrating and interdisciplinary meta-system ('Management') shown as a square, with CN's task being to observe and cross-fertilise problem domains, and observe the 'Environment'.

The $\mathrm{CN}$ discipline acts as a meta-system, investigates the $\mathrm{CN}$ problem domains and using attenuation mechanism, invokes the relevant terminology, models and theories from $\mathrm{CN}$-related disciplines (e.g. industrial engineering, management science, control engineering, information and communication technology,...) to respond to new issues arising in these domains.

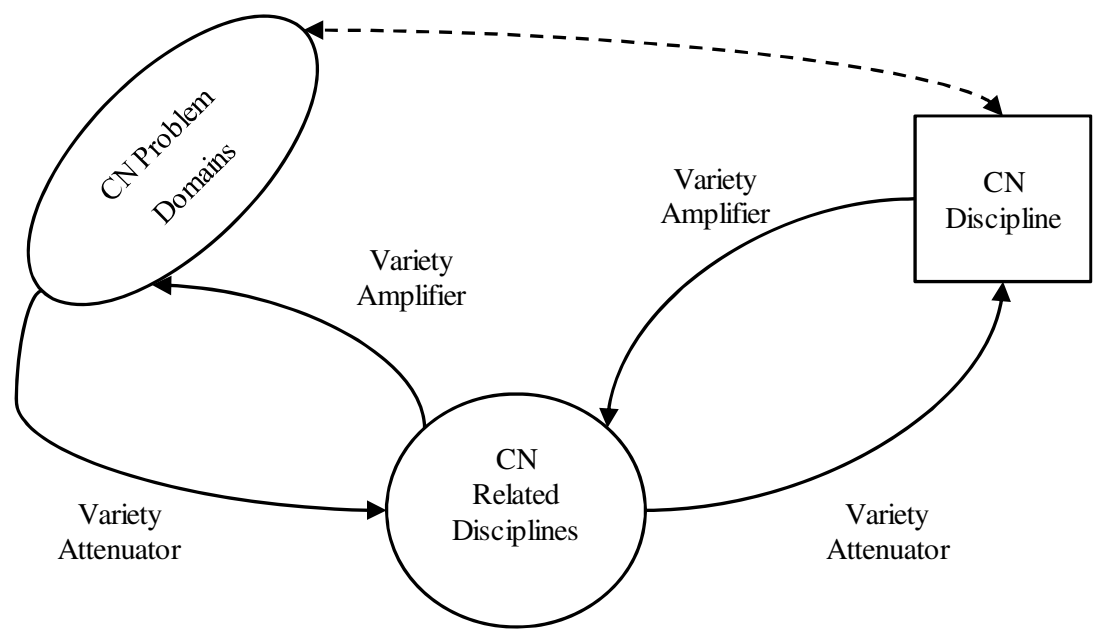

Fig. 1. Three components of a Viable CN Discipline

Changes in the problem domains mandate the evolution of individual $\mathrm{CN}$ related disciplines, so as to respond to the new requirements of the evolving environment.

In order to harmonise this co-evolution, we need to understand what the relevant mechanisms to guarantee an effective evolution of the $\mathrm{CN}$ discipline itself are.

If we consider the $\mathrm{CN}$ discipline as 'problem solving', then the step-by-step stages of co-evolution would be: 1) diagnose a significant problem in the $\mathrm{CN}$ problem domain, 2) invoke one or more relevant disciplines studying the $\mathrm{CN}$ problem domain and decide if such multi-disciplinary combined action is adequate, and if not, then 3 ) provide solutions for $\mathrm{CN}$ problems by harmonising and integrating multiple theories, models, techniques and methods from relevant disciplines in a synthesis (new or extended theory), and 4) adopt any 'new' case records of relevant disciplines and integrate them into the CN Body of Knowledge.

The need for a unifying theory clarifies the role of the $\mathrm{CN}$ discipline as a metasystem (as in Beer's VSM) answering a) what CN problem domains would be addressed in specific $\mathrm{CN}$ practices?, b) what would be the invoked disciplines 
targeting the problem domains in combined use?, c) how to formalise and harmonise other disciplines' contributions and apply them in a $\mathrm{CN}$ practice?

As the invoked disciples are continuously progressing and evolving in their specific domain and field of application, a more effective $\mathrm{CN}$ practice could be guaranteed if the evolution of these disciplines were influenced or monitored by the $\mathrm{CN}$ discipline and the findings reflected in $\mathrm{CN}$ theory and practice when necessary.

\section{Co-evolution Mechanisms for an Evolving CN Discipline}

We discussed three components of a viable $\mathrm{CN}$ discipline in Section 2, now the question arises: what are the mechanisms to keep the requisite variety of the $C N$ discipline as a viable system?

\subsection{Co-evolution Path Model, Dynamic Homeostasis vs. Dynamic Hetereostasis}

Beer [12] argues that a key property of a viable system and a "measure of its submission to the control mechanism" is its ability to maintain its equilibrium or homeostasis, which he defines as "constancy of some critical variables (outputs)". In our model of co-evolution, we define the dynamic sustenance of requisite variety based on Ashby's law: "only variety can destroy variety" [11], paraphrased by Beer [16] as "variety absorbs variety". Here, 'variety' is the number of possible states of a system [17], or as recently re-interpreted and refined by Kandjani and Bernus [18], the number of relevant states of a system.

Considering the system and its environment as two coupled entities, if one component is perturbed, the effect of that perturbation on the other component is either amplified through positive feedback, or may be reversed (attenuated) through negative feedback. These channels serve as self-perpetuating mechanisms of the system. (Note that what we call here a 'system' includes the system's controller.)

The role of the negative feedback loop is to reverse the effect of the initial perturbation and restore the system's homeostasis (in which critical variables are stable), while positive feedback can create unstable states [19].

As both the system and its environment (including systems in that environment) evolve, change can create imbalance between the requisite variety of our system of interest and the variety that would be required for it to maintain homeostasis. In other words, systems that want to live long must co-evolve with their environment.

More formally: we consider the environment an entity with a possible set of observable states and if two such states require different response from the system then the system must be able to differentiate between them (thus they are two different relevant states). Consequently in Fig. 2, for this purpose, the complexity of a system (CS) is defined to be the complexity of the model that the controller of the system maintains (appears to be maintaining) in order to manage the system's operations, so as to maintain adequate interaction with the environment. 
The complexity of the system's environment (CE) is a relative notion and is defined to be the complexity of the model of the environment that the controller of the system would need to maintain the in system's homeostasis. Specifically, such an 'environment model' must have predictive capability, so that the system, while interoperating with the environment, could maintain a homeostatic trajectory.

These models are needed to be able to represent and predict the states of signals and resources among the system, the external systems and the rest of the environment. This because based on the theorem of the 'Good Regulator' [20], a good controller of a system must have a model of that system with an equal complexity at its disposal as the system to be controlled has.

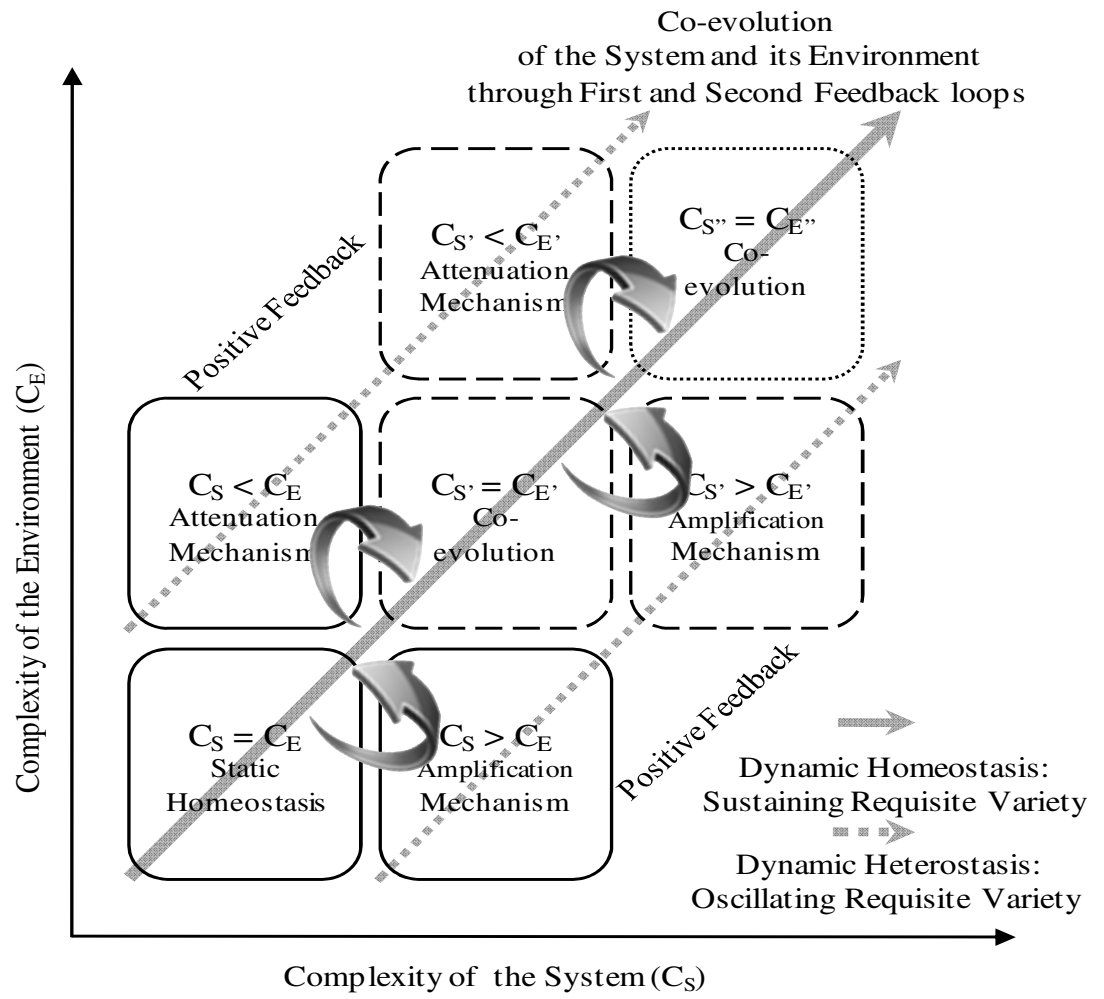

Fig. 2. Co-evolution Path Model

1) If the complexity of the system (CS) equals to that of its environment (CE), then the system has the requisite variety and is in static equilibrium. However, any change in the complexity of the environment should be sensed by the system's selfperpetuating mechanism to restore the system to its initial state or to create a new equilibrium state.

2) If the complexity of the environment is greater than that of the system, then the system should attenuate the effects of this complexity, i.e., change and co-evolve with its environment (in other words, the environment produced, or is recognised to have the potential to produce, some states in which the system cannot function adequately). 
3) If the complexity of the system is greater than that of its environment, then the system can potentially create a set of different states and perform behaviours, which are not differentiated by its environment. The system can identify this extra complexity as undesired, or use an amplification mechanism to create new differentiations in the environment.

Any system has a number of variables characterizing its essential survival properties. Ashby refers to these as 'essential variables', and defines survival as: “a line of behaviour [that] takes no essential variable outside given limits" [21,22] . Therefore, by definition, any line of behaviour outside limits of essential variables is on the non-viable system path and is fatal to the system.

For a system to be regarded as adaptive, and therefore viable, Ashby introduces two necessary feedback loops [21,22,23]. The first loop makes small modifications and corrections to the system. As opposed to this, the second loop changes the structure or architecture of the system and operates if essential variables are predicted to fall outside the limits of survival. If the system's second feedback loop does not respond to the changes in complexity of the environment, then the system will be on a non-viable path.

Based on Ashby's theory of adaptation [21], according to Umpleby [23], the first feedback loop is necessary for a system to learn a pattern of behaviour necessary for a specific environment, while the second feedback loop is required for a system to identify the changes in the environment and design/create new patterns of behaviour. If there is a dramatic increase in environment complexity and the system is not prepared to act, then the lack of functioning of the second feedback loop makes the system non-viable and the system is doomed to fail.

\subsection{Co-evolution Path Model of the CN Discipline}

Looking at the $\mathrm{CN}$ discipline as a system ( 'discipline-as-a-system') the co-evolution model of Section 3.1 applies to that system too (see Fig. 3), therefore the question is: what are the co-evolution mechanisms through which the $C N$ discipline can maintain requisite variety and remain relevant in light of changes to problem domain changes?

The $\mathrm{CN}$ discipline as an integrating discipline invokes models, theories, and methods of related disciplines, and effective co-evolution is only guaranteed by: a) invoking the right theories, models, and methods from $\mathrm{CN}$-related Disciplines (CNRD) to address new and emerging CN Problem Domains (CNPD) in a combined use (attenuation mechanism), and b) promoting new synthesised $\mathrm{CN}$ terminologies, reference models, and methods to provide solutions in $\mathrm{CN}$ problem domains using a holistic approach (amplification mechanism).

Thus, if at any one time the variety of the unified theory of $\mathrm{CN}$ discipline is less than the variety of the $\mathrm{CN}$ problem domains, then the $\mathrm{CN}$ discipline cannot respond to the needs of the environment problem domain) and must increase its variety by attenuating the relevant variety (e.g. adopt new elements from relevant $\mathrm{CN}$ related disciplines). On the other hand, a CN researcher or practitioner should also formulate and execute a 'promotion' mechanism if the variety of $\mathrm{CN}$ models, methods and frameworks is more than the variety of the $\mathrm{CN}$ problem domains. In this case, system 
managers, users, and stakeholders would not be able to comprehend these complex $\mathrm{CN}$ models, methods and etc. and would probably avoid using them, therefore one should decrease the variety of these models, or promote the use of more complex models by inventing new applications in the problem domain.

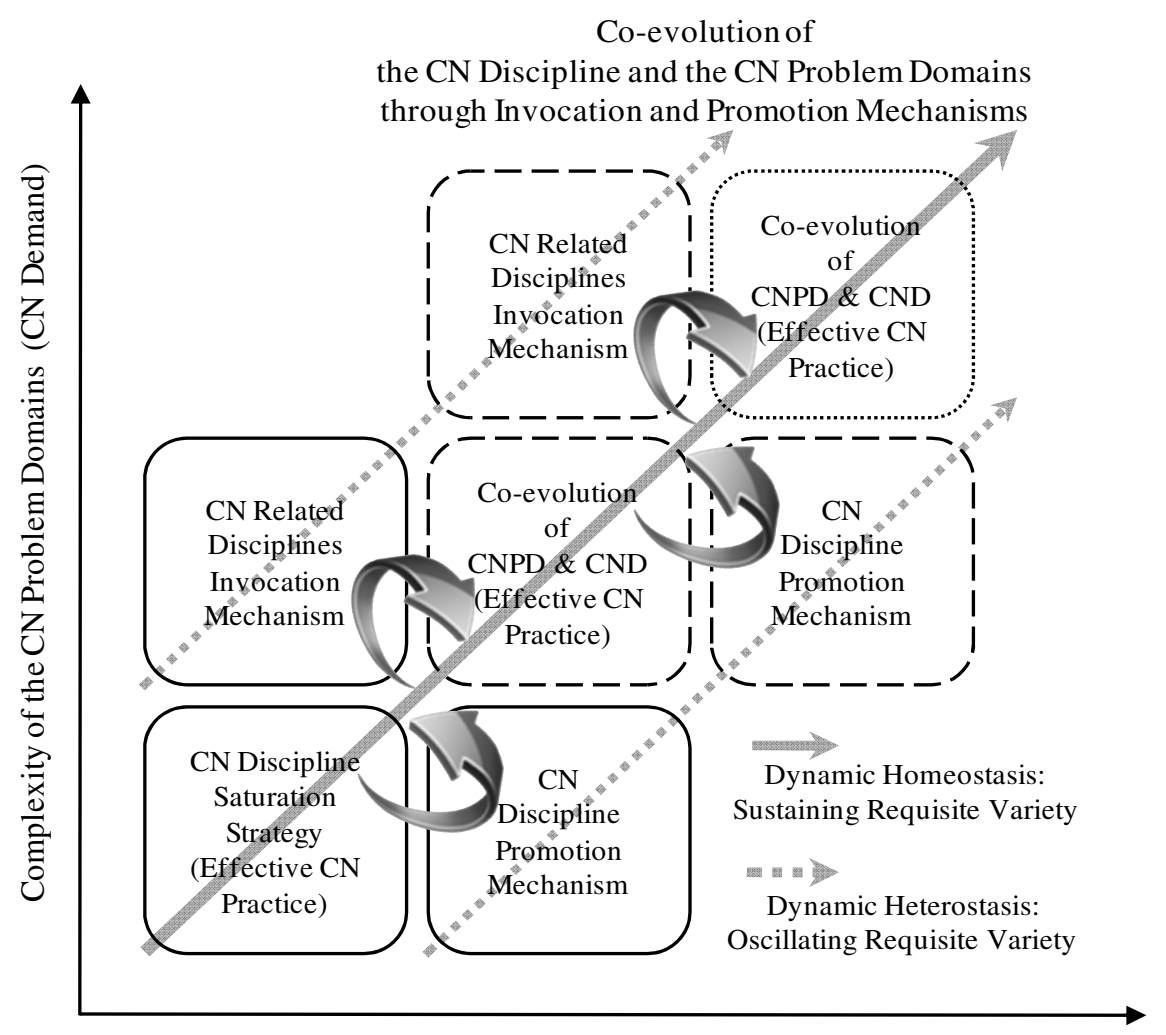

Complexity of the Collaborative Networks Discipline (CN Supply)

Fig. 3. Co-evolution Path Model of the Collaborative Networks Discipline

The $\mathrm{CN}$ discipline as an integrating discipline invokes models, theories, and methods of related disciplines, and effective co-evolution is only guaranteed by: a) invoking the right theories, models, and methods from $\mathrm{CN}$-related Disciplines (CNRD) to address new and emerging CN Problem Domains (CNPD) in a combined use (attenuation mechanism), and b) promoting new synthesised CN terminologies, reference models, and methods to provide solutions in $\mathrm{CN}$ problem domains using a holistic approach (amplification mechanism).

Thus, if at any one time the variety of the unified theory of $\mathrm{CN}$ discipline is less than the variety of the $\mathrm{CN}$ problem domains, then the $\mathrm{CN}$ discipline cannot respond to the needs of the environment problem domain) and must increase its variety by attenuating the relevant variety (e.g. adopt new elements from relevant $\mathrm{CN}$ related 
disciplines). On the other hand, a $\mathrm{CN}$ researcher or practitioner should also formulate and execute a 'promotion' mechanism if the variety of $\mathrm{CN}$ models, methods and frameworks is more than the variety of the $\mathrm{CN}$ problem domains. In this case, system managers, users, and stakeholders would not be able to comprehend these complex $\mathrm{CN}$ models, methods and etc. and would probably avoid using them, therefore one should decrease the variety of these models, or promote the use of more complex models by inventing new applications in the problem domain.

The evolution of problem domains must therefore be closely monitored (including strategic forecasts) so as to be able to perform the mentioned 'invocation' and 'promotion' to provide the $\mathrm{CN}$ problem domains with relevant combined disciplinecontributions in practice.

\section{Conclusions}

To develop a theory of evolution of the $\mathrm{CN}$ Body of Knowledge, we focused on the viability of the $\mathrm{CN}$ discipline as a system, discussed it using Beer's Viable System Model (VSM), and introduced its three components using VSM. We also proposed the concept co-evolution mechanisms concept for the evolving $\mathrm{CN}$ discipline based on VSM and a companion theory, Ashby's law of requisite variety, but used a new complexity measure, that takes the relativity of this term into account.

Our cybernetic model of the evolution of the $\mathrm{CN}$ discipline enriches Anderton and Checkland's model of developing disciplines, who previously demonstrated the cyclic interaction between theory development and formulation for a problem, and theory testing [24,25], warning about the need for theories to remain 'relevant' (viable). Our model which is based on the Co-evolution Path Model [26,27] now illustrates the mechanisms of theory development from this point of view.

A relevant field of study called 'Enterprise Architecture Cybernetics (EAC)' $[28,29,30]$ has a similar purpose and level of abstraction to this intended theory, however EAC has a different scope and genericity (namely its scope encompasses all socio-technical systems of systems in the broadest sense, including social, economic and ecological systems, and aggregates thereof).

Future work will concentrate on applying this model, whereupon testing and validation of this theoretical model of discipline development is to be performed by illustrating the above effects through concrete cases from the development of the $\mathrm{CN}$ body of knowledge-hopefully also discovering new, open problems in this domain.

\section{References}

1. Kandjani, H., Bernus, P.: Towards a Cybernetic Theory and Reference Model of Self designing Complex Collaborative Networks. In: Camarinha-Matos, L.M., Xu, L., Afsarmanesh, H. (eds.) Collaborative Networks in the Internet of Services. IFIP AICT, vol. 380, pp. 485-493. Springer, Heidelberg (2012) 
2. Camarinha-Matos, L.M., Afsarmanesh, H. (eds.): Collaborative Networked Organizations, A Research Agenda for Emerging Business Models. Kluwer Academic Publishers, Boston (2004)

3. Camarinha-Matos, L.M., Afsarmanesh, H.: A roadmap for strategic research on virtual organizations. In: Processes and Foundations for Virtual Organizations. Kluwer Academic Publishers, Boston (2003)

4. Camarinha-Matos, L.M., Afsarmanesh, H.: The emerging discipline of collaborative networks. In: Virtual Enterprises and Collaborative Networks. Kluwer Academic Publishers, Boston (2004)

5. Camarinha-Matos, L.M., Afsarmanesh, H.: Collaborative networks: A new scientific discipline. Journal of Intelligent Manufacturing 16(4), 439-452 (2005)

6. Camarinha-Matos, L.M., Afsarmanesh, H.: On reference models for collaborative networked organizations. Int. J. of Production Research 46(9), 2453-2469 (2008)

7. Camarinha-Matos, L., Afsarmanesh, H.: Towards a reference model for collaborative networked organizations. In: Shen, W. (ed.) Information Technology for Balanced Manufacturing Systems. IFIP, vol. 220, pp. 193-202. Springer, Boston (2006)

8. Camarinha-Matos, L., Afsarmanesh, H.: Motivation for a theoretical foundation for collaborative networks. In: Collaborative Networks: Reference Modeling, pp. 5-14. Springer, Berlin (2008)

9. Camarinha-Matos, L.M., Afsarmanesh, H., Ollus, M.: ECOLEAD: A holistic approach to creation and management of dynamic virtual organizations. In: Camarinha-Matos, L.M., Afsarmanesh, H., Ortiz, A. (eds.) Collaborative Networks and their Breeding Environments. IFIP, vol. 186, pp. 3-16. Springer, Boston (2005)

10. Wiener, N.: Cybernetics or Control and Communication in the Animal and the Machine (2nd Rev. Ed. 1961) (1961); MIT Press, Cambridge (1948)

11. Ashby, W.R.: An introduction to cybernetics. Chapman \& Hall, London (1956)

12. Beer, S.: Decision and Control: The Meaning of Operational Research and Management Cybernetics. Wiley, New York (1966)

13. Beer, S.: Diagnosing the system for organizations. Wiley, New York (1985)

14. Boulding, K.E.: General systems theory-the skeleton of science. Management Science 2(3), 197-208 (1956)

15. Bertalanffy, L.: General System Theory-Foundations and Developments. George Braziller, Inc., New York (1968)

16. Beer, S.: The Heart of Enterprise: The Managerial Cybernetics of Organization. Wiley, New York (1979)

17. Beer, S.: Brain of the Firm, 2nd edn. Wiley, New York (1981)

18. Kandjani, H., Bernus, P.: Engineering Self-Designing Enterprises as Complex Systems Using Extended Axiomatic Design Theory. IFAC Papers On Line 18(1), 11943-11948 (2011)

19. Ashby, W.R.: Adaptiveness and equilibrium. The British Journal of Psychiatry 86(362), 478-483 (1940)

20. Conant, R.C., Ashby, W.R.: Every Good Regulator of a System Must be a Model of That System. International Journal of Systems Science 1(2), 89-97 (1970)

21. Ashby, W.R.: Design for a brain; the origin of adaptive behavior. Wiley, New York (1960)

22. Geoghegan, M.C., Pangaro, P.: Design for a self-regenerating organisation. International Journal of General Systems 38(2), 155-173 (2009)

23. Umpleby, S.A.: Ross Ashby's general theory of adaptive systems. International Journal of General Systems 38(2), 231-238 (2009) 
24. Anderton, R.H., Checkland, P.B.: On learning our lessons. Internal Discussion Paper. Lancaster, UK, Department of Systems, University of Lancaster. 2/77 (1977)

25. Checkland, P.: Systems Thinking, Systems Practice. Wiley, Chichester (1996)

26. Kandjani, H., Bernus, P., Nielsen, S.: Co-evolution path model: How enterprises as complex systems survive on the edge of chaos. In: ACIS 2012: Location, location, location: Proceedings of the 23rd Australasian Conference on Information Systems, pp. 1-11 (2012)

27. Kandjani, H., Bernus, P., Nielsen, S.: Enterprise Architecture Cybernetics and the Edge of Chaos: Sustaining Enterprises as Complex Systems in Complex Business Environments. In: Proceedings of the 46th Hawaii International Conference on System Sciences, HICSS 2013, pp. 3858-3867. IEEE (2013)

28. Kandjani, H., Bernus, P.: Capability Maturity Model for Collaborative Networks Based on Extended Axiomatic Design Theory. In: Camarinha-Matos, L.M., Pereira-Klen, A., Afsarmanesh, H. (eds.) PRO-VE 2011. IFIP AICT, vol. 362, pp. 421-427. Springer, Heidelberg (2011)

29. Kandjani, H., Bernus, P.: Evolution of the Enterprise Architecture Discipline: Towards a Unified Developing Theoryof EA. In: Proc. ICEIS 2012, Warsaw, pp. 145-154 (2012)

30. Kandjani, H., Wen, L., Bernus, P.: Enterprise Architecture Cybernetics for Collaborative Networks: Reducing the Structural Complexity and Transaction Cost via Virtual Brokerage. Information Control Problems in Manufacturing 14(1), 1233-1239 (2012) 